\title{
Analytical Algorithm Expressions in Simulation of the Temperature Field in Electric Resistance Spot Welding
}

\author{
Miomir VUKIĆEVIĆ, Mišo BJELIĆ, Dragan MILČIĆ, Miroslav MIJAJLOVIĆ, Marina PLJAKIĆ
}

\begin{abstract}
The paper presents the method of obtaining mathematical equations named "Analytical algorithm expressions" which enable simple creation of computer programs for simulation of the temperature field in the weld zone in electric resistance spot welding. Knowledge of these equations and the manner of their formulation make creation of program packages easier but they do not change anything with respect to the structure and scope of necessary input data which determine concrete initial and boundary conditions. In addition to providing algorithm description of the temperature field, the considered approach is applied for mathematical description of the field of any other physical value relevant for the welding process (specific current, electric potential, density, etc.). In this paper they are realized in temperature fields, as hierarchically superior to the fields of stress and current density, i.e. fields of physical properties of materials of the sheet and the electrode (superiority refers to the algorithm domain). Results of simulation for the non-stationary period of the welding process at two extreme discreet moments are presented at the end.
\end{abstract}

Keywords: analytical algorithm expressions; electric resistance spot welding; iterative procedure; finite differences; non-stationary part of the process; simulation; stationary part of the process; temperature field

\section{INTRODUCTION}

Electric resistance spot welding is a welding process where the heat, which is needed to weld metal pieces together, is generated by applying an electric current through the sheets which are pressed between the electrodes [1].

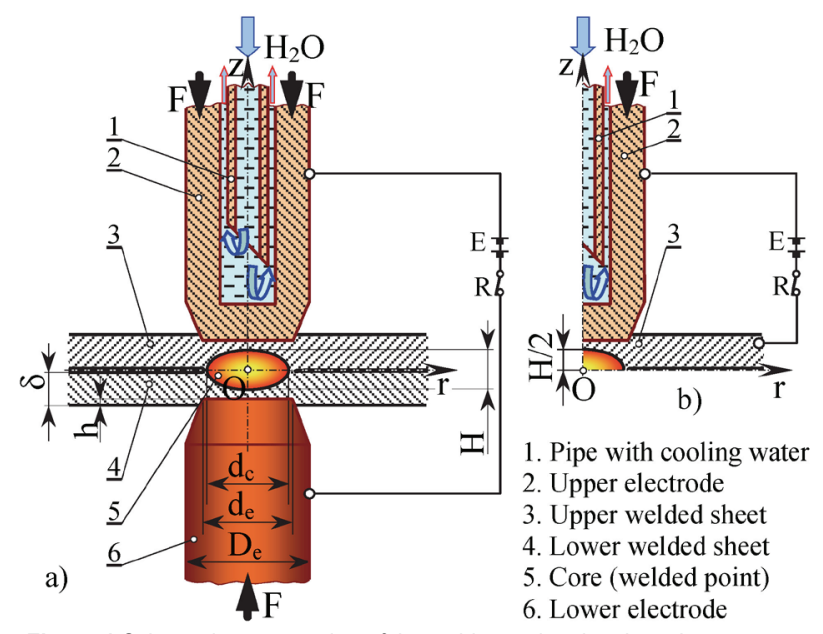

Figure 1 Schematic presentation of the weld zone by electric resistance spot welding

In electric resistance spot welding, the temperature fields as well as distribution of other physical values are considered in the geometrical space presented in Fig. 1a. The physical axial symmetry of that space in relation to the $\mathrm{r}$ and $\mathrm{z}$ axes is shown. Therefore, all analyses of the process are carried out in one quadrant of the welding zone (b), making conscious deviation from the reality in which only the upper electrode is cooled (it is mainly movable). The weld zone is considered in the cylindrical coordinate system. Electric resistance spot welding is made of three main sub processes/phenomena, Fig. 2.

Simulation modelling of welding processes gives us possibility to better understand influences of main phenomena. First analytical models of spot welding $[2,3]$ showed up in 1960's. The application of numeri- cal models started in 1980's with development of computers [4]. Success and significance of the welding model is even greater if it covers a large number of the mentioned phenomena.Today's models consider temperature dependent material properties, phase transformations and coupled thermal, electrical and mechanical interactions $[5,6]$.

The electric process covers all the pertaining physical phenomena and all elements of the electric circuit which starts at the connection of the machine to the power supply network, continues by transmission of current through the control elements and the transformer and ends at the electrodes and welded sheets. The mechanical process is based on the application of pressure force before, during and after the existence of the current flow and covers the consequences which arise.

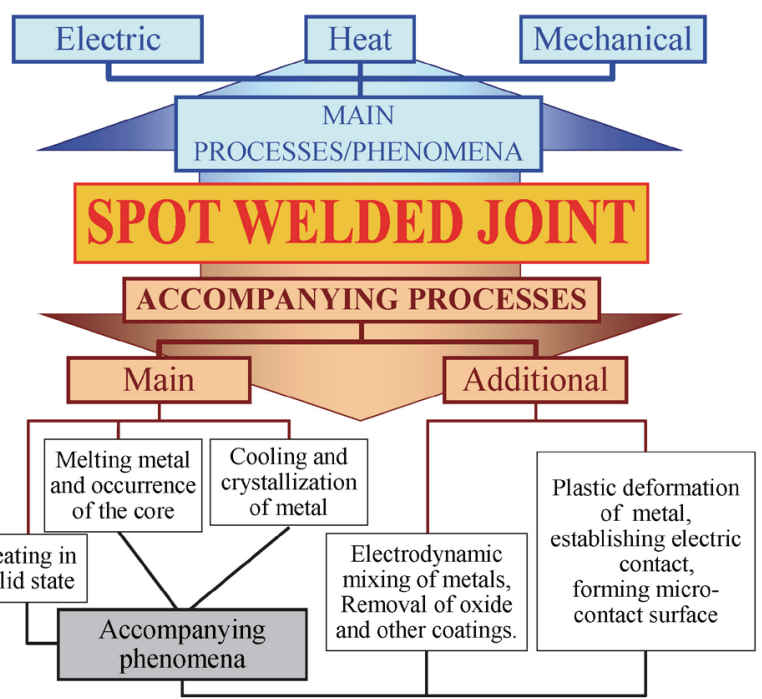

Figure 2 Complementary macro processes in spot welding and their structure

The algorithm structure of the process of determination of temperature fields in welded sheets and electrodes is made of two logically and structurally similar procedures. The first procedure refers to the initial period of welding (stationary), and the second one refers to all 
other discrete moments of the periods of welding and cooling (non-stationary).

Homogeneity of the fields of all physical properties of materials of the sheet and the electrode at the moment which precedes establishing the electric field $(t=0-\varepsilon)$, i.e. in the first passage through the program package is assumed. That assumption is valorised by procedures of determination of the corresponding fields $(\rho, S, \lambda)$. In the non-stationary period of welding $(t>0)$, that block denotes the procedures of calculation of discrete values of the fields with the same physical properties $(\rho, S, \lambda)$.

In relation to the stationary conditions of calculation, the procedures which belong to the non-stationary period are characterized by extreme temperature dependence of all properties of material of the sheet and the electrode, i.e. voltage and current, which makes the main difference between these two periods. The period of cooling the sheet and the electrode starts at the moment of interruption of electric current flow.

The "Exact theoretical calculation" [7], has not been successfully and completely developed so far since it is still impossible to include changes and descriptions of all present processes, phenomena and states of real material medium in analytical calculations and models. For solving formulated partial differential equations, based on the application of computers, this paper presents realization of the numerical method of finite differences - iterative procedure. That is why the calculation grid whose cells have different shapes is formed in the weld zone, in accordance with Fig. 3. The elementary plan-polygons are formed by connecting central points (nodes) of four or less than four neighbouring cells, depending on the position of the central cell in the considered geometrical space and in accordance with the corresponding boundary conditions.

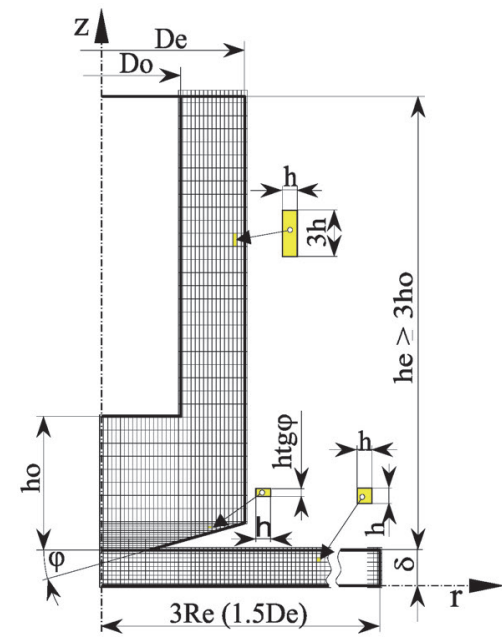

Figure 3 Geometrical discretization of the weld zone in the form of the variable calculation grid, [8]

On the other hand, coinciding of physical boundaries of the area with the position of central points (nodes) of boundary layers of the cells is provided. In all directions, the number of cells is an integer value. For the purpose of as real modelling of geometry of the weld zone as possible, the variable step grid - multigrid method is applied.
The motion strategy (iterative procedure) is in accordance with the Gauss-Seidel method for line. The adopted strategy respects natural tendencies of development of each analysed process in the corresponding quadrant.

For the needs of simulation of the non-stationary part of the welding process, upon discretization of the geometrical space (by the coordinates $i, j$ ), discretization by the time $t$ starts. The main problem is expressed in determination of the time increment $\Delta t$, i.e. time step between two discrete moments of the process. Two values are obtained by applying the same methodology of determination of the time increment.

The solution, described in this paper, was accomplished by two times discretization. The first one refers to the space of the welded sheet. In non-stationary conditions of the process, the sheet area is simulated by one discrete step of time. Discretization of the electrode space is realized by the second discrete step. These two values depend on each other.

The accomplished geometrical discretization of the weld zone space imposes procession of over 6500 systems of algebraic equations in each iteration and at every discrete moment.

\section{CALCULATION GRID}

The realized variable calculation grid, shown in Fig. 3 , was created as a consequence of three noticed geometrical areas which approximate the considered quadrant. In the first space, in the zone of welded sheets, the cells of square shape with the discrete step $h$ are applied. The conical ending of the electrode (working part) is approximated by the grid of rectangular cells with the discrete step $h$ in the direction of the $r$-axis, i.e. $k$ in the $z$ direction:

$k=h_{z}=h \cdot \tan \varphi$

The rest of the electrode space (cylindrical part in which there is a coolant hole) is also covered by a rectangular grid. The sides of its cells, in the $\mathrm{z}$-direction, i.e. the corresponding discrete step, have the value:

$k=h_{z}=3 h$

This is done because electric and heat processes are realized at a lower energy level so that the credibility of description ( $3 h)$ is not lost by a large step. Application of the variable calculation grid made the developed program solutions more complex. The main problem is seen in the necessity of finding procedures for moving from the grid with a finer increment to the grid with a rougher geometrical increment, and vice versa.

The solutions described in literature refer to elimination of errors of big wave lengths, and in this case they are inapplicable, at least not for approximation of key physical values. The final solutions are realized based on the principle of the law of conservation of energy and properties of superposition expressed in the elliptic type of second order differential equations with constant coef- 
ficients. They were realized by implementation of fictive nodes on the basis of linear interpolation.

\section{ANALYTICAL DESCRIPTION OF THE TEMPERATURE FIELD}

Establishing and maintenance of the variable temperature field of the considered continuum is expressed by the gradient:

$\operatorname{grad} T=\nabla T=\vec{i} \cdot \frac{\partial T}{\partial x}+\vec{j} \cdot \frac{\partial T}{\partial y}+\vec{k} \cdot \frac{\partial T}{\partial z} \equiv \vec{n}_{0} \cdot \frac{\partial T}{\partial n}$

The specific heat flow is obtained by reducing the total heat flow $Q$ to the considered section in the unit of time:

$\overrightarrow{\dot{q}}=\vec{q}=-\left(\vec{n}_{0}\right) \cdot \frac{\partial Q}{\partial S} \cdot \frac{1}{d t}$

The specific heat flux, i.e. the flow is reduced to the unit of cross section by conduction, Eq. (5), convection, Eq. (6) and radiation, Eq. (7):

$\overrightarrow{\dot{q}}=\vec{q}=-\lambda \cdot \nabla T=-\lambda \cdot \vec{n}_{0} \cdot \frac{\partial T}{\partial n}$

$\dot{q}=q=\alpha \cdot\left(T_{z}-T_{f}\right)$

$\dot{q}=C_{s} \cdot \frac{S^{2}}{\pi \cdot z^{2}} \cdot\left[\left(\frac{T_{x}}{100}\right)^{4}-\left(\frac{T_{0}}{100}\right)^{4}\right]$

Very frequently, and even in this paper, a more suitable form of the previous expression is used:

$\dot{q}_{z}=a_{z} \cdot S \cdot\left(T_{x}-T_{0}\right)$

Based on Eqs. (7) and (8), the coefficient of heat transfer by radiation is determined by the equation:

$\alpha_{z}=C_{S} \cdot \frac{\left(T_{x}\right)^{4}-\left(T_{0}\right)^{4}}{T_{x}-T_{0}} \cdot \frac{S}{\pi \cdot z^{2}} \cdot 10^{-8}$

The general expression for transformation of electric into heat energy, in the conductor of invariable section $\mathrm{S}$, during the time $t$, reads:

$\dot{q}_{e l}=r \cdot j^{2} \cdot l \cdot S$

\subsection{Electric Process}

From the aspect of function in the analytical model of spot welding, three parameters of the electric process are characteristic:

\section{a) Electrical resistance}

By their physical nature and place of existence, Fig. 4, there are two different types of electrical resistance:
- natural or volume electrical resistance, $R_{\mathrm{d}}$ and $R_{\mathrm{e}}$,

- contact or superficial electrical resistance, $R_{\text {ed }}$ and $R_{\mathrm{dd}}$.
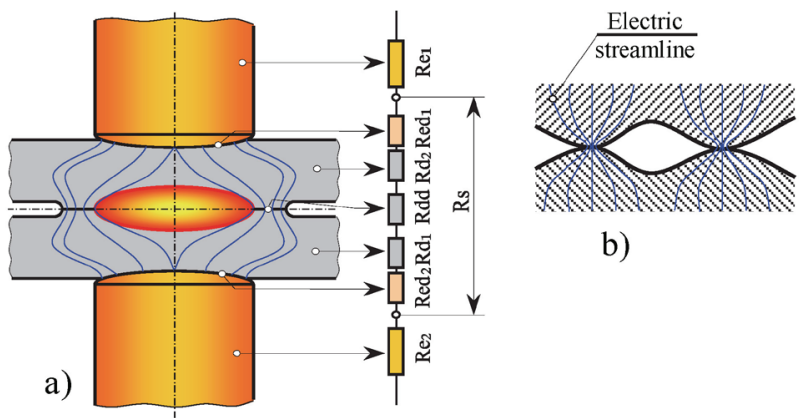

b)

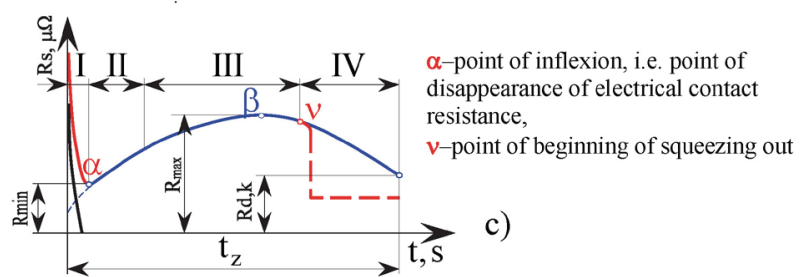

Figure 4 The characteristic values of the electrical resistance in the welding zone: a) position of partial resistance and the equivalent electrical scheme, b) physical and contact micro relief, c) dynamical curve of changes of the electrical resistance.

In addition to the type of conductive medium, the value of electrical resistance is significantly influenced by temperature and geometry of the conductive medium (its length $\ell$ and the cross section $\mathrm{S}$ ).

\section{b) Potential of the electric field - voltage}

The general differential equation of the field of potential in the conditions of uniform temperature field (Laplace), and hence in the conditions of uniform field of specific volume electrical resistance, $[9,10]$, has the form:

$\frac{\partial}{\partial z}\left(\frac{1}{\rho} \cdot \frac{\partial \varphi}{\partial z}\right)+\frac{\partial}{\partial r}\left(\frac{1}{\rho} \cdot \frac{\partial \varphi}{\partial r}\right)+\frac{1}{r} \cdot \frac{1}{\rho} \cdot\left(\frac{\partial \varphi}{\partial r}\right)=0$

By transformation of each member of the general equation of approximation of the potential field into finite differences, it reads:

$\frac{1}{\rho_{0}} \cdot\left(\varphi_{1}+\varphi_{2}+\varphi_{3}+\varphi_{4}-4 \cdot \varphi_{0}+\frac{\varphi_{3}-\varphi_{1}}{2 \cdot i}\right)+\frac{1}{4} \cdot$
$\cdot\left[\left(\frac{1}{\rho_{3}}-\frac{1}{\rho_{1}}\right) \cdot\left(\varphi_{3}-\varphi_{1}\right)+\left(\frac{1}{\rho_{2}}-\frac{1}{\rho_{4}}\right) \cdot\left(\varphi_{2}-\varphi_{4}\right)\right]=0$

By solving the previous equation, the value of potential at the central point of the polygon shown in Fig. 5 is determined by applying the following expression:

$\varphi_{0}=\frac{1}{4}\left(\phi_{1}+\phi_{2}+\phi_{3}+\phi_{4}+\frac{\phi_{3}-\phi_{1}}{2 \cdot i}\right)+\frac{\rho_{0}}{16}$.

$\cdot\left[\left(\frac{1}{\rho_{3}}-\frac{1}{\rho_{1}}\right) \cdot\left(\phi_{3}-\phi_{1}\right)+\left(\frac{1}{\rho_{2}}-\frac{1}{\rho_{4}}\right) \cdot\left(\phi_{2}-\phi_{4}\right)\right]$ 
The criterion for completion of the iterative procedure, i.e. the level of allowed deviation, $\varepsilon$ :

$$
\left|\varphi_{0}^{(k)}-\varphi_{0}^{(k-1)}\right| \leq \varepsilon
$$

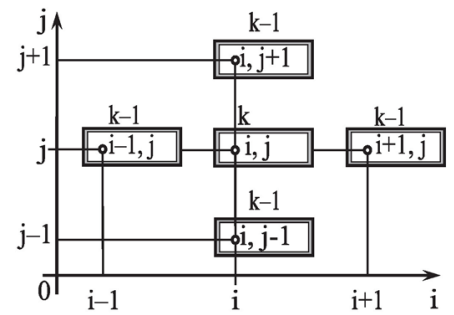

Figure 5 General representation of the method of central pattern (elementary plan-polygon)

If the temperature field, at the considered moment of the welding process, can be regarded as uniform, and the specific electrical resistance $\rho(r, z)=$ const., which is one of the characteristics of the initial period of welding, the equilibrium state of potential is described by the linear second order partial differential equation, of the elliptic type:

$\frac{\partial^{2} \varphi}{\partial z^{2}}+\frac{\partial^{2} \varphi}{\partial r^{2}}+\frac{1}{r} \cdot \frac{\partial \varphi}{\partial r}+0$

The equation of approximation of electric potential, for stationary conditions, is obtained when each member of the previous equation is expressed in the form of finite differences, so that its developed form reads:

$\varphi_{0}=\frac{1}{4} \cdot\left(\varphi_{1}+\varphi_{2}+\varphi_{3}+\varphi_{4}+\frac{\varphi_{3}-\varphi_{1}}{2 \cdot i}\right)$

\section{c) Density of welding current (specific)}

The voltage drop between any two points in the conductor with invariable section, in the stationary field, is proportional to the strength of current which passes through it. For the non-stationary electric field it is not possible to establish equations of voltage equivalence and potential difference, so that it is necessary to generalize:

$j=\frac{1}{\rho} \cdot \frac{\partial \varphi}{\partial \ell}$

In the cylindrical coordinate system, the potential difference $(\partial \varphi)$ is different in different directions ( $z$ and $r$ ), so that the corresponding components of the vector of current density read:

$$
\begin{aligned}
& j_{z}=\frac{1}{\rho_{0}} \cdot \frac{\varphi_{z+1}-\varphi_{z-1}}{2 \cdot k} \\
& j_{r}=\frac{1}{\rho_{0}} \cdot \frac{\varphi_{r+1}-\varphi_{r-1}}{2 \cdot h}
\end{aligned}
$$

I.e. the summed current density $j_{0}$, at every element of the calculation grid, is determined as a module of the vector sum of the components:

$j_{0}=\sqrt{j_{z}^{2}+j_{r}^{2}}$

When numerical denotations of nodes of the calculation grid are applied Fig. 5, the general expression for determination of current density in the node $0^{\prime}$ of the central layout is obtained:

$$
j_{0}=\frac{1}{2 \cdot \rho_{0}} \cdot \sqrt{\left(\frac{\varphi_{2}-\varphi_{4}}{k}\right)^{2}+\left(\frac{\varphi_{3}-\varphi_{1}}{h}\right)^{2}}
$$

\subsection{Heat Process}

The energy balance of the process (heat balance) results from the law of conservation of energy:

$\sum_{i=1}^{i=n} \dot{q}_{i}=0 ; \quad\left(\dot{q}+\dot{q}^{\prime}\right)+\dot{q}^{\prime \prime}=0$

The exchanged amount of heat in the considered elementary volume, at the time $d t$ is equal to:

$\dot{q}=(-\lambda) \cdot\left(\frac{\partial^{2} T}{\partial r^{2}}+\frac{1}{r} \cdot \frac{\partial T}{\partial r}\right) \cdot d V+(-\lambda) \cdot \frac{\partial^{2} T}{\partial z^{2}} \cdot d V$

The accumulated heat in the elementary volume during the time $d t$ :

$\dot{q}=C \cdot \gamma \cdot \frac{\partial T}{\partial t} \cdot d V$

The heat release due to the existence of an internal heat source in the elementary volume during the time $d t$ :

$\dot{q}^{\prime}=q_{i} \cdot d V \equiv q_{e l}$

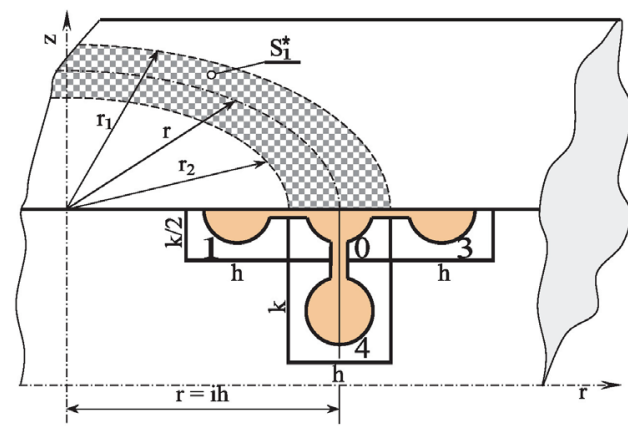

Figure 6 Schematic representation of analytical establishing of heat balance for the elementary layer in the cylindrical coordinates [11]

The equation of distribution of temperatures is obtained by replacing the previous three expressions in Eq. (22) and by its rearranging on the basis of graphical interpretation of the process of heat exchange in the cylindrical coordinate system, Fig. 6 : 
$a \cdot\left(\frac{\partial^{2} T}{\partial z^{2}}+\frac{\partial^{2} T}{\partial r^{2}}+\frac{1}{r} \cdot \frac{\partial T}{\partial r}\right)+\frac{q_{i}}{C \cdot \gamma}=\frac{\partial T}{\partial t}$

\section{a) Stationary temperature field}

In the mathematical sense, the stationary temperature field is defined by the absence of the member on the right-hand side of the equals sign in Eq. (26), i.e. $\partial T / \partial t=0$. It means that in the weld zone there is no accumulated energy and that the temperature field does not depend on time but only on coordinates. In the weld zone there is an infinite number of heat sources, and the heat flow of each of them, on the basis of Joule law, is expressed by the following equation:

$$
q_{i}=\frac{q_{e l}}{V}=j^{2} \cdot \rho, \mathrm{W} / \mathrm{m}^{3}
$$

By replacing the obtained expression for the flow of the heat source in Eq. (27), for the stationary conditions, it is obtained that:

$$
\frac{\partial^{2} T}{\partial z^{2}}+\frac{\partial^{2} T}{\partial r^{2}}+\frac{1}{r} \cdot \frac{\partial T}{\partial r}+\frac{q_{i}}{\lambda}=0
$$

The numerical method of solving this equation based on approximation of solution by finite differences consists of substitution of partial derivatives by the corresponding finite differences:

$$
\begin{aligned}
& \frac{\partial^{2} T}{\partial z^{2}}=\frac{1}{k^{2}} \cdot\left(T_{i, j+1}-2 \cdot T_{i, j}+T_{i, j-1}\right) \\
& \frac{\partial^{2} T}{\partial r^{2}}=\frac{1}{h^{2}} \cdot\left(T_{i+1, j}-2 \cdot T_{i, j}+T_{i-1, j}\right) \\
& \frac{1}{r} \cdot \frac{\partial T}{\partial r}=\frac{1}{h \cdot i} \cdot \frac{1}{2 h} \cdot\left(T_{i+1, j}-T_{i-1, j}\right)
\end{aligned}
$$

The induced amount of heat of the internal heat source is invariant in relation to the applied coordinate system (coordinates/position), and therefore the last member of Eq. (28), in its unchanged form, is contained in its solution. The differential form is obtained by replacing the Eqs. (29), (30), (31) and it reads:

$$
\begin{array}{r}
\frac{1}{k^{2}} \cdot\left(T_{i, j+1}+T_{i, j-1}\right)+\frac{2 i+1}{2 \cdot i \cdot h^{2}} \cdot T_{i+1, j}+ \\
+\frac{2 i-1}{2 \cdot i \cdot h^{2}} \cdot T_{i-1, j}-\frac{h^{2}+k^{2}}{h^{2} \cdot k^{2}} \cdot T_{i, j}=0
\end{array}
$$

If approximation by finite differences is realized by means of the quadrant calculation grid, for the central and neighbouring cells, taking into account Eq. (28), the recurrence iterative equation for determination of temperature in the central node is obtained in the form:

$$
\begin{aligned}
T_{i, j}= & \left(\frac{1}{4} \cdot T_{i, j+1}+T_{i, j-1}+\frac{2 i+1}{2 \cdot i} \cdot T_{i+1, j}+\right. \\
& \left.+\frac{2 i-1}{2 \cdot i} \cdot T_{i-1, j}+\frac{j_{i, j}^{2} \cdot \rho_{i, j}}{\lambda_{i, j}} \cdot h^{2}\right)
\end{aligned}
$$

\section{b) Non-stationary temperature field}

The equilibrium state of the non-stationary temperature field at any discrete moment, for the time increment $\Delta t$ which precedes it, is described by Eq. (26). It is obvious that the stationary temperature field represents its special case.

By replacing Eqs. (29-31) and taking into account Eq. (27), the recurrence iterative equation for iterative procedure of approximation of Eq. (26) is obtained, using the finite difference method:

$$
\begin{aligned}
T_{i, j}= & \frac{1}{1+F_{o}} \cdot\left[T_{i, j}^{(k-1)}+F_{o} \cdot\left(T_{i, j+1}+T_{i, j-1}+\right.\right. \\
& \left.\left.+\frac{2 i+1}{2 \cdot i} \cdot T_{i+1, j}+\frac{2 i-1}{2 \cdot i} \cdot T_{i-1, j}+T_{u}\right)\right]
\end{aligned}
$$

The denotations of positions of nodes $(i, j)$ are in accordance with Fig. 5. The "stationary" part of Eq. (34) is noticed in its structure (in parentheses), which is explained in the identical way as the left-hand side of Eq. (26). The "stationary" part defines the equilibrium state of the temperature field for the current time increment by using the superposition property of the finite difference method. Eq. (34) is convergent. This statement is true if the so-called method of right-hand derivatives with any time and geometrical increment is applied for its solving. Of course, a higher level of precision of equilibrium state of the temperature field is realized if smaller values of increments are adopted. All other values of temperatures, except $T_{i, j}^{(k-1)}$ are taken from the set of values from the current or previous iteration, but at the current discrete moment.

If the method of left-hand derivatives were applied, it would be necessary, depending on the boundary conditions, to test the criterion of stability (convergence) for every equation from the obtained system of equations. In Eq. (34), the time domain of the temperature field is determined by the Fourier dimensionless number:

$F_{o}=\frac{\lambda \cdot \Delta t}{\gamma \cdot C \cdot h^{2}}$

Similarly to the stationary temperature field, the temperature increment in the central node, as a consequence of existence of an internal heat source, for the square calculation grid, is determined by the expression:

$T_{u}=\frac{j_{i, j}^{2} \cdot \rho_{i, j}}{\lambda_{i, j}} \cdot h^{2}$ 


\section{Time Increment}

The coefficient of temperature conductivity a, $\mathrm{m}^{2} / \mathrm{s}$, i.e. the Fourier dimensionless number $F_{o}$, Eq.(35) determines the kinetics of heat exchange between elementary layers in the continuum, i.e. after integration in the continuum as a whole (since the weld zone is considered as homogeneous and non-deformable medium).

For analytical and algorithm procession, the acceptable solution is obtained by mathematical analysis of Eq. (26), which is by [11], reduced to the form:

$$
\Delta t=\xi \cdot \frac{\gamma \cdot C}{4 \cdot \lambda} \cdot h^{2}, \quad \xi=\frac{x}{2 \sqrt{a \cdot t}}
$$

where $\xi$ is the parameter of the Fourier function:

$$
F(\xi)=e^{-\xi^{2}}-\xi \cdot[1-G(\xi)]
$$

Since the Fourier number depends not only on the type of material and temperature but also on the characteristic of the calculation grid $h$, the algorithm provides the calculation of values of the Fourier number in all necessary conditions, which was the decisive factor in selection of the final solution of the methodology of determination of time increment for the non-stationary part of the process in the electrode zone. Hence the relative ratio $\tau$ between time increments for the sheet and the electrode was introduced, and its calculation is performed at every discrete moment:

$\tau=\frac{\Delta t_{L}}{\Delta t_{E}}$

Taking into account the properties of material of the sheet and the electrode at the initial moment of welding, the value of parameter of time increment is:

$$
\tau=4.7 \times 10^{-3} \cdot \frac{F_{o}^{L}}{F_{o}^{E}}
$$

In the developed program package, the relevant criterion for determination of the parameter of time increment represents the step of the calculation grid in the $z-$ direction $(k=h \cdot \tan \varphi)$.

\section{ANALYTICAL ALGORITHM EXPRESSIONS}

When the procedure of obtaining heat balance equations [8] as well as the structure of each of 33 developed equations are taken into account, depending on whether they refer to the stationary or non-stationary period of the welding process, two types of algorithm forms applied in creation of the computer program are established.

\subsection{Stationary Period of the Welding Process}

The heat balance equations of this type do not contain the member $\dot{q}^{\prime}$ from Eq. (22). The total of $33 \mathrm{SHBE}$ (stationary heat balance equations) was developed, and their general form, which is named the algorithm expression of heat balance equations, is expressed in the following way:

$$
\begin{aligned}
T_{0}= & \frac{1}{K_{A}+K_{z}+\sum_{i=1}^{4} K_{i}} \cdot\left[\sum_{i=1}^{4}\left(K_{i} \cdot T_{i}\right)+\right. \\
& \left.+K_{A} \cdot T_{A}+K_{x} \cdot T_{z}+T_{u}\right]
\end{aligned}
$$

The equation contains variables and their indices with the following meanings:

\subsection{Non-Stationary Period of the Welding Process}

Eq. (34) represents the general form of equations which make the algebraic system of every plan-polygon. If the denotations of the pertaining nodes (cells) are as in Fig. 3 and Fig. 5 and when the members which express heat exchange by convection (in continuation it is divided into natural and forced convection) and radiation are included in Eq. (34), its somewhat simpler form is obtained:

$$
\begin{aligned}
T_{0} & =\frac{1}{F_{o}+1} \cdot\left[T_{0}^{*}+F_{o} \cdot K_{o}\left(K_{1} T_{1}+K_{2} T_{2}+K_{3} T_{3}+\right.\right. \\
& \left.\left.+K_{4} T_{4}+K_{X} T_{X}+K_{z} T_{z}+T_{u}\right)\right]
\end{aligned}
$$

Boundary conditions have a crucial influence on the structure of Eq. (42). Therefore, the obtained equations differ not only by the presence or absence of certain members, but also by the structure of each of the mentioned coefficients/multi-pliers $(K)$. Besides, the member before the square brackets $\left(1 /\left(F_{o}+1\right)\right)$ is also a characteristic of similar influences.

For the unique physical medium (it is not homogeneous, but the one without geometrical and matter discontinuity), i.e. for the plan - polygons distributed in internal parts of the sheet area and the electrode area (their central nodes are not at the boundaries of contact), the following general analytical algorithm expression is formulated:

$$
\begin{aligned}
T_{0}= & \frac{1}{1+F_{o} \cdot K_{o} \cdot\left(K_{A}+K_{z}+\sum_{i=1}^{4} K_{i}\right)} \cdot\left\{T_{0}^{*}+\right. \\
& \left.+F_{o} \cdot K_{o} \cdot\left[\sum_{i=1}^{4}\left(K_{i} \cdot T_{i}\right)+K_{A} \cdot T_{A}+K_{z} \cdot T_{z}+T_{u}\right]\right\}
\end{aligned}
$$

In the second case, characteristic by its referring to the central cells distributed along the contacts electrodesheet, the general analytical algorithm expression reads:

$$
\begin{aligned}
T_{0}= & \frac{1}{1+K_{A}+\sum_{i=1}^{4} K_{i}} \cdot\left[T_{0}^{*}+\sum_{i=1}^{4}\left(K_{i} \cdot T_{i}\right)+\right. \\
& \left.+K_{A} \cdot T_{A}+T_{u}\right]
\end{aligned}
$$


The seemingly simpler structure of Eq. (44) is characterized by the expressions for coefficients $K_{i}$ which are considerably more complex than the corresponding ones in Eq. (43). It is because Eq. (44) describes, besides other things, the change of the physical medium so that the corresponding Fourier dimensionless numbers are contained in their expressions. The transformed form of the product $\gamma \cdot C$ from Eq. (35) is used:

$\gamma^{*} C^{*}=\gamma_{\mathrm{L}} C_{\mathrm{L}}+\gamma_{\mathrm{E}} C_{\mathrm{E}} \cdot \tan \varphi-$ for the contact between the working surfaces of the electrode and the sheet,

$\gamma^{*} C^{*}=\gamma_{\mathrm{L}} C_{\mathrm{L}}+0.75 \gamma_{\mathrm{E}} C_{\mathrm{E}}-$ for the conical tip as a contact between the working surfaces of the cylindrical part of the electrode and the sheet.

The indices indicate the group of properties of materials of the sheet $(\mathrm{L})$ or the electrode $(\mathrm{E})$. The Fourier numbers have similar expressions as in Eq. (35), i.e.:

$F_{o L}^{*}=\frac{\lambda_{L} \cdot \Delta t}{\gamma^{*} \cdot C^{*} \cdot h^{2}}-$ for the electrode area,

$F_{o E}^{*}=\frac{\lambda_{E} \cdot \Delta t}{\gamma^{*} C^{*} \cdot h^{2}}-$ for the welded sheet area

The described analytical algorithm expressions represent the minimum form of Eq. (34), i.e. Eq. (42) and allow program (software) interpretation of all heat balance equations, i.e. identification of temperature fields in the physical area of the sheet and the electrode at every discrete moment of the welding process as a whole.

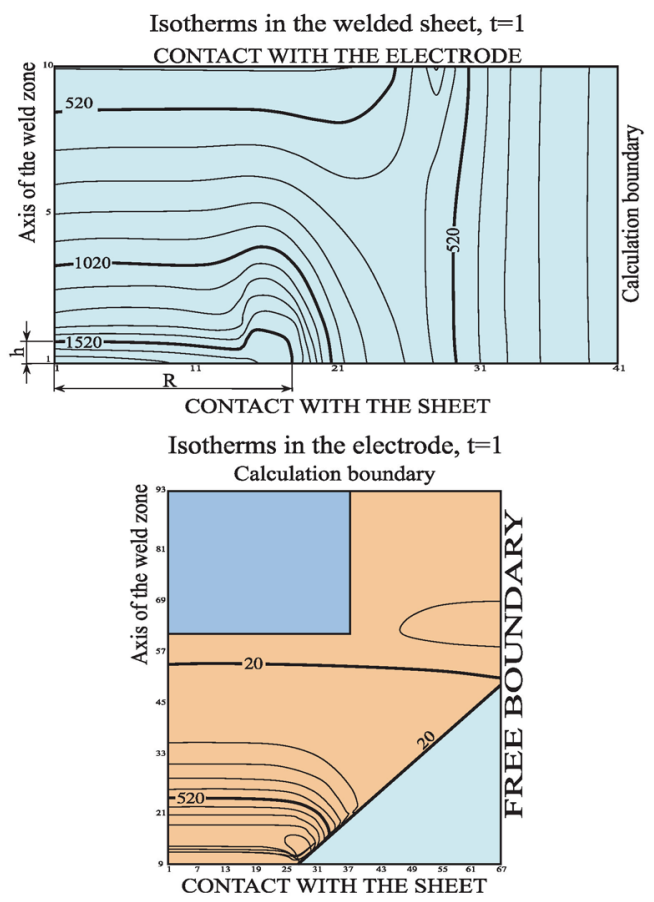

Figure 7 Isotherms in the weld zone at the first discrete momen

\section{PRESENTATION OF RESULTS}

The obtained general analytical algorithm expressions make development of computer programs easier, i.e. they are applied for software description of the previ- ously developed heat balance equations for the stationary and non-stationary welding periods $[8,2]$. Spatial distributions of all relevant physical values ending with the temperature field in the weld zone are obtained as software output. The examples of planar distribution are presented in Fig. 7 for non-stationary conditions of process development. The first one shows the isotherms of the temperature field at the first discrete moment of the non-stationary period, and in the second one they are at the boundary discrete moment (final). The temperature jump at the first discrete moment is the consequence of the initial temperature field and the existence of electrical contact resistance.

On the other hand, such temperature distribution, since it directly depends on the field of electric potential and electric current, is in accordance with the so-called skin effect, i.e. with the Greenwood concept of the model, [2], by which, in the initial period, the extreme values of current are achieved at the periphery of contact electrode-sheet and sheet-sheet.

\section{CONCLUSIONS}

Application of the developed program package for simulation of electric resistance spot welding $[10,4]$ and its results presented here show that it is necessary for the simulation model to include the electrode area. In a lot of papers it is not done because it is stated that the temperature field in the electrode does not change to a considerable extent. This paper does not deny that statement, but it shows that the influence of the electrode does exist and it is neither possible nor desirable to neglect it. Secondary iteration which simulates the process of heat exchange in the electrode area provides obtaining results that respect the use of cooling liquid. It allows taking away heat from the electrode area and maintaining the acceptable temperature distribution in the electrode. Actually, the electrodes are used for taking away heat even from the zone of welded sheets thus influencing the dimensions of the formed welded point. Application of analytical algorithm expressions makes the operation of computers faster, which creates the precondition for development of a simultaneous system of control of the subject process in which it is necessary that the simulation speed should be identical to the speed of the process itself. If another discretization of time is integrated in the simulation model, as it is presented in this paper, or in a similar way, it should be expected that the precision of simulation results will be increased. In the concrete case, the similarity of simulation values with the real (measured) ones reaches $90 \div 91.9 \%$, [8].

\section{Acknowledgement}

The authors wish to express their gratitude to the Ministry of Education and Science of the Republic of Serbia (project TR37020).

\section{REFERENCES}

[1] Zhang, H. \& Senkara, J. (2011). Resistance welding: fundamentals and applications, CRC press, Boca Raton Florida, 
[2] Greenwood, J. A. (1961). Temperatures in spot welding. British Welding Journal, 8(6), 316-322.

[3] Rice, W. \& Funk, E. J. (1967). An analytical investigation of temperature distributions during resistance welding. Welding journal, 46(4), S175.

[4] Nied, H. (1984). The finite element modeling of the resistance spot welding process. Welding journal, 63(4), 123.

[5] Shen, J., Zhang, Y., Lai, X. \& Wang, P. C. (2011). Modeling of resistance spot welding of multiple stacks of steel sheets. Materials \& Design, 32(2), 550-560. https://doi.org/10.1016/j.matdes.2010.08.023

[6] Huh, H. \& Kang, W. J. (1997). Electrothermal analysis of electric resistance spot welding processes by a 3-D finite element method. Journal of Materials Processing Technology, 63(1), 672-677. https://doi.org/10.1016/S0924-0136(96)02705-7

[7] Yamamoto, T. \& Okuda, T. (1972). Spot welding of heavy-gauge mild steel. Soud Techn Connexes, 26(1), 4356.

[8] Vukićević, M., Petrović, Z., Kolarević, M. \& Bjelić, M. (2009). Simulation model of initial period of spot welding. Mathematical Modeling of Weld Phenomena / Graz, 955966.

[9] Орлов, Б. Д., Чакалев, А. А. \& Дмитриев, Ю. В. (1986). Техно-логия и оборудование контактной сварки, Машино-строение, Москва.

[10] Чакалев, А. А. \& Прохоров, А. Н. (1989). Совершенствование термодеформационной модели контактной точечной сварки. Сварочное производство, 4, 29-32.

[11] Кочергин, К. А. (1987). Контактная сварка, Машиностроение, Москва.

\section{Nomenclature}

\begin{tabular}{|ccc|}
\hline$C$ & - & the specific heat (heat capacity), $\mathrm{J} / \mathrm{kg}^{\circ} \mathrm{C}$ \\
$h$ & - & the step of the discrete grid in the direction of the $r$-axis, \\
$i_{y}$ & - & discrete coordinates \\
$k$ & - & ordinal number of iteration \\
$\dot{q}$ & - & the exchanged amount of heat in the considered elemen- \\
$\dot{q}^{\prime}$ & & tary volume \\
$\dot{q}^{\prime \prime}$ & - & the accumulated heat in the elementary volume during \\
$r$ & - & the heat release due to the existence of an internal heat \\
$S$ & - & the radius, i.e. position of the cell in the direction of the \\
$T_{1}$ & - & the characteristic area of the heat conductive fibre, $\mathrm{m}^{2}$ \\
$T_{0}$ & - & the temperature in the central cells of the polygon, ${ }^{\circ} \mathrm{C}$ \\
$\Delta t$ & - & the time increment, $\mathrm{s}$ \\
$\lambda$ & - & the coefficient of thermal conductivity, $\mathrm{W} /\left(\mathrm{m}^{\circ} \mathrm{C}\right)$ \\
$\rho$ & - & the specific electrical conductivity, $\Omega \mathrm{m}$ \\
$\varphi$ & - & the angle of inclination of the electrode cone generatrix, \\
$\alpha$ & - & the coefficient of heat transfer $($ convection $), \mathrm{W} /\left(\mathrm{m}^{2} \mathrm{~K}\right)$ \\
$\gamma$ & - & the density of the physical medium, $\mathrm{kg} / \mathrm{m}^{3}$ \\
& &
\end{tabular}

\section{Contact information:}

Miomir VUKIĆEVIĆ, Associate Professor

Faculty of Mechanical and Civil Engineering in Kraljevo

University of Kragujevac

Dositejeva 19, 36000 Kraljevo, Serbia

E-mail: vukicevic.m@mfkv.kg.ac.rs

Mišo BJELIĆ, Teaching Assistant

Faculty of Mechanical and Civil Engineering in Kraljevo University of Kragujevac

Dositejeva 19, 36000 Kraljevo, Serbia

E-mail: bjelic.m@gmail.com

\section{Dragan MILČIĆ, Full Professor}

Faculty of Mechanical Engineering

University of Niš

Aleksandra Medvedeva 14, 18000 Niš, Serbia

E-mail: milcic@masfak.ni.ac.rs

Miroslav MIJAJLOVIĆ, Assistant Professor

Faculty of Mechanical Engineering

University of Niš

Aleksandra Medvedeva 14, 18000 Niš, Serbia

E-mail: mijajom@masfak.ni.ac.rs

Marina PLJAKIĆ, PhD Student

Faculty of Mechanical and Civil Engineering in Kraljevo University of Kragujevac

Dositejeva 19, 36000 Kraljevo, Serbia

E-mail: pljakic.m@mfkv.kg.ac.rs 